This item was submitted to Loughborough's Research Repository by the author.

Items in Figshare are protected by copyright, with all rights reserved, unless otherwise indicated.

\title{
Models incorporating pin joints are suitable for simulating performance but unsuitable for simulating internal loading
}

PLEASE CITE THE PUBLISHED VERSION

http://dx.doi.org/10.1016/j.jbiomech.2012.02.019

PUBLISHER

(C) Elsevier

VERSION

AM (Accepted Manuscript)

LICENCE

CC BY-NC-ND 4.0

\section{REPOSITORY RECORD}

Allen, Samuel J., Mark A. King, and Maurice R. Yeadon. 2019. "Models Incorporating Pin Joints Are Suitable for Simulating Performance but Unsuitable for Simulating Internal Loading". figshare.

https://hdl.handle.net/2134/10029. 
This item was submitted to Loughborough's Institutional Repository (https://dspace.lboro.ac.uk/) by the author and is made available under the following Creative Commons Licence conditions.

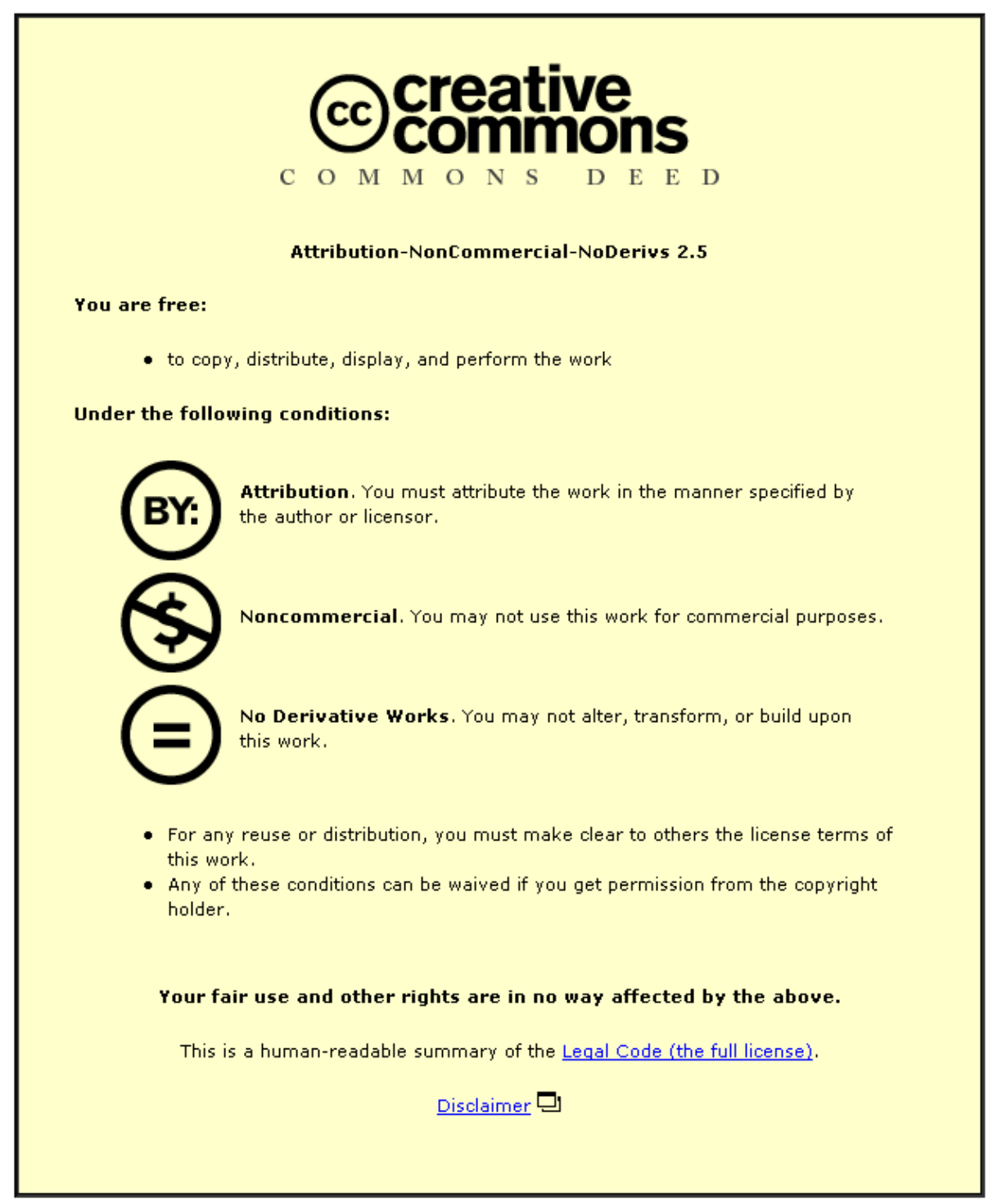

For the full text of this licence, please go to: http://creativecommons.org/licenses/by-nc-nd/2.5/ 


\title{
Models incorporating pin joints are suitable for simulating performance but unsuitable for simulating internal loading
}

\author{
S.J. Allen, M.A. King, and M.R. Yeadon \\ School of Sport, Exercise, and Health Sciences, Loughborough University, LE11 3TU, UK
}

\begin{abstract}
Simulation models of human movement comprising pin-linked segments have a potential weakness for reproducing accurate ground reaction forces during high impact activities. While the human body contains many compliant structures such a model only has compliance in wobbling masses and in the foot-ground interface. In order to determine whether accurate GRFs can be produced by allowing additional compliance in the footground interface, a subject-specific angle-driven computer simulation model of triple jumping with 13 pin-linked segments was developed, with wobbling masses included within the shank, thigh, and trunk segments. The foot-ground interface was represented by spring-dampers at three points on each foot: the toe, ball, and heel. The parameters of the spring-dampers were varied by a genetic algorithm in order to minimise the differences between simulated GRFs, and those measured from the three phases of a triple jump in three conditions: (a) foot spring compression limited to $20 \mathrm{~mm}$; (b) this compression limited to $40 \mathrm{~mm}$; (c) no restrictions. Differences of $47.9 \%, 15.7 \%$, and $12.4 \%$ between simulation and recorded forces were obtained for the $20 \mathrm{~mm}, 40 \mathrm{~mm}$, and unrestricted conditions respectively. In the unrestricted condition maximum compressions of between $43 \mathrm{~mm}$ and $56 \mathrm{~mm}$ were obtained in the three phases and the mass centre position was within $4 \mathrm{~mm}$ of the actual position at these times. It is concluded that the unrestricted model is appropriate for simulating performance whereas the accurate calculation of internal forces would require a model that incorporates compliance elsewhere in the link system.
\end{abstract}

Keywords: computer simulation, ground reaction forces, triple jump

\section{Introduction}

Ground reaction forces (GRFs) are amongst the most important measures in studies of human motion. Aside from direct measurement, GRFs can be estimated using theoretical models. In theory, inverse dynamics models can be used in order to calculate GRFs using whole body centre of mass (COM) accelerations but in practice GRFs are typically measured and used as input along with kinematic data for inverse dynamics calculations. Forward dynamics models commonly calculate GRFs by simulating collisions between the feet and the floor (Otten, 2003). When constructing simulation models of the human body, segments are often represented as rigid and pin-linked (Alexander, 1990; van Soest et al., 1993). Realistic GRFs have been reproduced by rigid pin-linked simulation models using springs to model ground contact during walking (Anderson and Pandy, 2001) and running (Neptune et al., 2000). Whilst this approach gives a reasonable approximation for activities which do not involve large impacts with the ground, it is unable to provide accurate representations of internal forces and torques when simulating high impact activities (Gruber et al., 1998). In order to reproduce realistic ground reaction forces in pinlinked simulation models of jumping, a requirement for a high level of compliance (70 $\mathrm{mm}$ ) at the foot-ground interface has been reported and attributed to a lack of wobbling masses (Seyfarth, 2000). Wobbling masses have been incorporated within forward dynamics models in an attempt to account for the shock attenuation properties of soft tissue during impacts (Gruber et al., 1998; Pain and Challis, 2004). 
Few studies using whole body simulation models incorporating wobbling masses have attempted to match recorded GRFs in high impact activities (King et al., 2006).

In addition to soft tissue which has been represented by wobbling masses in simulation models, the human skeletal system also contains compliance in the form of tissues such as ligaments and cartilage, which attenuate shock in the joints of the leg and spine (Chu et al., 1986; Hoshino and Wallace, 1987), and possibly in the bending of the long bones of the leg (Radin and Paul, 1970). As a consequence it might be expected that including wobbling masses within a pin-linked whole body model may not provide sufficient compliance for a high impact activity.

With this in mind, this study will investigate whether permitting increased levels of compliance at the foot-ground interface will allow pin-linked whole body computer simulation models to reproduce accurate GRFs. Simulating GRFs in the triple jump (Figure 1) represents a unique challenge, since it involves the highest measured vertical GRFs of any sporting activity (Hay, 1993; Perttunen et al., 2000).

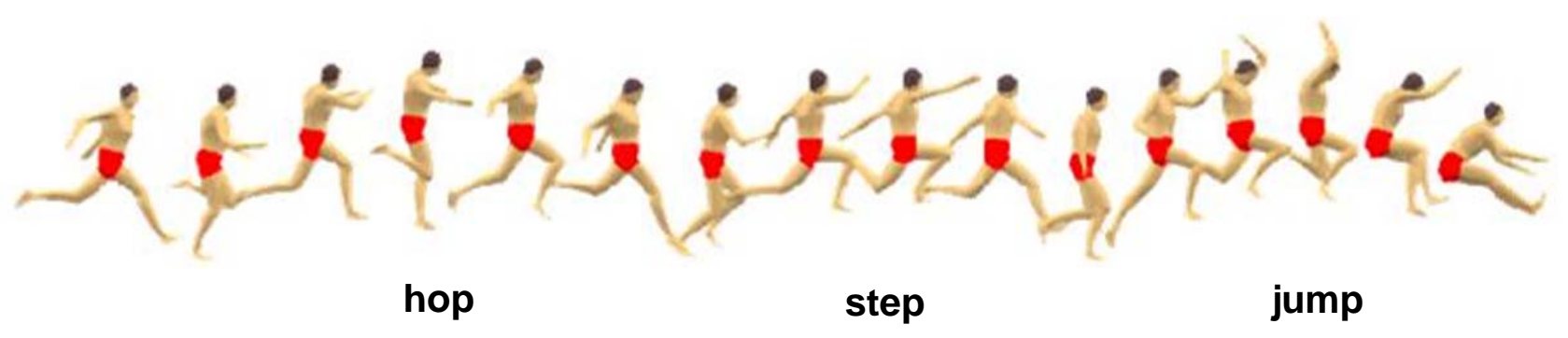

Figure 1. Graphics sequence of a triple jump.

\section{Methods}

Kinematic and force data were gathered at the Loughborough University indoor High Performance Athletics Centre (HiPAC) from a male triple jumper of national standard (age 22 years; mass 72.6 kg; height $1.82 \mathrm{~m}$; best performance $14.35 \mathrm{~m}$ ). The study was carried out in accordance with the Loughborough University Ethical Advisory Committee guidelines. Forty-five $25 \mathrm{~mm}$ retroreflective markers were placed in positions on the body of the subject in order that locations of joint centres could be calculated. Eighteen Vicon MX cameras covered a volume of $18 \mathrm{~m} \times 2 \mathrm{~m} \times$ $2.5 \mathrm{~m}$ spanning the last stride of the approach and the full triple jump. The subject was asked to perform the ground contact of each phase of the triple jump from a single force plate, necessitating three trials. Kinematic data were collected at 480 $\mathrm{Hz}$, and force data collected from an AMTI BP600900 force platform sampling at $1000 \mathrm{~Hz}$. Orientation of the trunk in a global reference frame, and joint configuration angles were calculated by projecting the joint centre coordinates onto the sagittal plane. The angle data were fitted with quintic splines (Wood and Jennings, 1979) for input to the simulation model using error estimates calculated as the difference between a data value and the mean of adjacent values as in Yeadon and King (2002). Ninety-five anthropometric measurements were taken and used as input to the inertia model of Yeadon (1990) in order to calculate subject-specific segmental inertia parameters. Centre of mass (COM) velocity at touchdown was calculated using COM position at takeoff and touchdown of the flight phases preceding each ground contact and was used as input to the model.

A 13-segment planar angle-driven computer simulation model of the ground contact phases of triple jumping (Figure 2) was constructed using Autolev ${ }^{\mathrm{TM}}$ (Kane and Levinson, 1996). The 13 segments represented: trunk + head, two upper arms, two forearms and hands, two thighs, two shanks, two 2-segment feet, with wobbling 
masses within the shanks, thighs, and trunk. Each foot had three points of contact with the ground at the heel, ball (metatarsophalangeal joint), and toe.

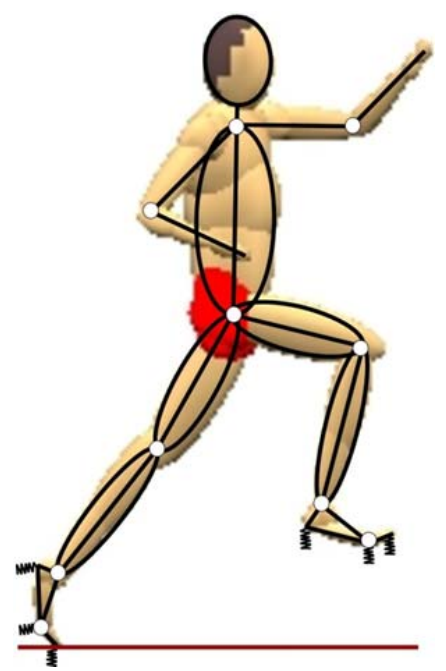

Figure 2. Thirteen-segment simulation model with wobbling masses within the shank, thigh, and trunk segments, angle drivers at all joints (white circles), and spring-dampers at three points on each foot.

The foot-ground interface was modelled using horizontal and vertical non-linear spring-dampers situated at the heel, ball, and toe of each foot:

$$
\begin{aligned}
& R_{z}=\sum_{i=1}^{3}-\left(k_{1 i} \cdot z_{i}+k_{2 i} \cdot z_{i}^{2}\right)-k_{3 i} \cdot \dot{z}_{l} \cdot\left|z_{i}\right|, \\
& R_{x}=\sum_{i=1}^{3}\left[-\left(k_{4 i} \cdot x_{i}+k_{5 i} \cdot x_{i}^{2}\right)-k_{6 i} \cdot \dot{x}_{i} \cdot\left|x_{i}\right|\right] \cdot R_{z i},
\end{aligned}
$$

where $R_{x}$ is the horizontal force, $R_{z}$ is the vertical force, $x$ and $z$ are the horizontal and vertical displacements, $\dot{x}$ and $\dot{z}$ are the time derivatives of $x$ and $z ; k_{1}, k_{2}, k_{4}, k_{5}$ are stiffness coefficients, $k_{3}, k_{6}$ are damping coefficients, and $i$ represents the point of contact on the foot.

The horizontal spring-damper expression was multiplied by the vertical force to ensure that the resulting horizontal force decayed to zero at takeoff. Each damping term incorporated the magnitude of the displacement in order that a force due to damping could not exist without displacement. The toe and ball spring-dampers shared the same parameters since both represented points on the spike plate of the athlete's shoe, whilst the heel differed.

Non-linear spring-dampers connected the ends of the wobbling and fixed elements of a segment (Pain and Challis, 2001):

$$
\boldsymbol{R}_{i}=\left(-k_{7 i} \cdot\left|\boldsymbol{r}_{i}\right|^{3}-k_{8 i} \cdot\left|\dot{\boldsymbol{r}}_{l}\right|\right) \cdot \hat{\boldsymbol{r}}_{i}, \quad \text { for } i=1,3
$$

where $\boldsymbol{R}$ is a force vector, $\boldsymbol{r}$ is a vector defining the position of the point of attachment on the wobbling mass from that on the fixed link, $\hat{\boldsymbol{r}}$ is a unit vector in the direction of $\boldsymbol{r},|\boldsymbol{r}|$ is the magnitude of $\boldsymbol{r},|\boldsymbol{r}|$ is the derivative of $|\boldsymbol{r}|, k_{7}$ and $k_{8}$ are stiffness and damping coefficients respectively, and $i$ represents the segment containing the wobbling mass.

A common set of viscoelastic parameters representing the attachments of the wobbling masses and the foot-ground interface was determined for the three phases of the triple jump using an adaptation of the method of Wilson et al. (2006). The 
simulation model was angle-driven using recorded kinematics and 27 parameters were varied using a genetic algorithm (GA) (Carroll, 1996) in order to minimise an objective function representing the difference between the simulation GRFs and measured GRFs horizontally $(x)$ and vertically $(z)$. These 27 parameters comprised: 12 stiffness and damping coefficients at the foot, six stiffness and damping coefficients for the wobbling masses, and nine kinematic parameters specifying the three initial angular and linear velocities of the whole body in each of the three phases. The objective function was composed of the percentage RMS differences between simulation and performance in: time to peak force, magnitude of peak force, and overall RMS difference between the force time histories. The objective function value $S$ was taken to be the average function value from each of the three ground contact phases of the triple jump in order that a robust common set of parameters was obtained (Wilson et al., 2006; Allen et al., 2010):

$$
S=\frac{1}{3} \sum_{i=1}^{3} R M S\left[f x_{i}, f z_{i},\left(\frac{t x_{i}+m x_{i}}{2}\right),\left(\frac{t z_{i}+m z_{i}}{2}\right)\right],
$$

where $f x$ and $f z$ are the RMS differences between the force time histories expressed as a percentage of peak force, $t x$ and $t z$ are the percentage differences in time to peak force, $m x$ and $m z$ are the percentage differences in the magnitude of peak force, and $i$ represents the phase of the jump.

Often GA-generated simulations with high stiffness values at the foot-ground interface would lead to the foot breaking and re-making contact with the ground. To prevent this from happening, takeoff was considered to have occurred if all points on the foot broke contact with the ground at any time during the stance phase. Differences between experiment and simulation RMS of force time histories were calculated for the greater of either the recorded ground contact time or the ground contact time of the simulation. Therefore a simulation that took off prematurely would incur a large RMS difference between the force time histories. This acted to encourage the GA to generate simulations with stiffness and damping parameters that led to ground contact times that were similar to recorded times.

Penalties were incurred if wobbling mass COM displacement exceeded what were considered reasonable limits of $50 \mathrm{~mm}, 75 \mathrm{~mm}$, and $100 \mathrm{~mm}$ at the shanks, thighs, and trunk respectively (Lafortune et al., 1992; Minetti and Belli, 1994). Penalties were added to the objective function value at a weighting of $1 \%$ for every $10 \mathrm{~mm}$ that the maximum excursion of each wobbling mass COM exceeded the limit. No penalties were incurred for wobbling mass movement in the optimised solutions.

Three matching optimisations were run: one where the compression in the springs representing the foot-ground interface was restricted in both the horizontal and vertical direction to $20 \mathrm{~mm}$; one where it was restricted to $40 \mathrm{~mm}$; and one where it was unrestricted. $20 \mathrm{~mm}$ was chosen as an estimate of realistic maximum vertical compression in the foot-ground interface; this comprised $10 \mathrm{~mm}$ compression in the heel pad calculated for an applied force of $10 \mathrm{kN}$ (representing the maximum measured GRF in this study) using the equation of Pain and Challis (2001), and 10 $\mathrm{mm}$ compression in the sole of the shoe and the ground (Alexander and Bennett, 1989). The total level of compression at the foot-ground interface in the actual performance was found to be $19 \mathrm{~mm}$ from the change in the vertical position of the ankle joint centre from the point of impact of the heel to the point at which the ankle joint centre was at its minimum vertical position. Horizontally the same limits were applied, although it was not expected that they would be reached, since it was 
imagined that the compression requirements were much greater vertically than horizontally.

The movement of the feet was restricted by stringent ramped penalties which were added to the objective function value using the following weighting:

$$
P=\sum_{i=1}^{2} 10^{8} \cdot\left(d_{i}-l\right)^{2}, \text { for } d_{i}>l
$$

where $P$ is the percentage value added to the objective function, $l$ is the limit in metres, $d$ is the maximum displacement of any point on the foot in metres, and $i$ represents the direction (horizontal or vertical) of the displacement.

The ability of the foot-ground interface representation to provide realistic centre of pressure locations is crucial since errors will result in a different moment of vertical force producing errors in whole body rotation. In the matching simulations the trunk angle was one of the degrees of freedom and was not included in the objective difference function and so provided an independent measure of this aspect of model performance. The RMS difference between recorded and simulated trunk angle was calculated.

A comparison of mass centre vertical position between recorded performance and simulation at the time of maximum vertical depression was made by double integrating the vertical acceleration calculated from the recorded force to determine mass centre movement.

\section{Results}

When penalties restricting the depression of the foot-ground interface to $20 \mathrm{~mm}$ were employed, the optimisation process was unable to match recorded forces well (Figure 3), yielding objective function values representing differences of $44.3 \%$, $55.5 \%$, and $43.9 \%$ between simulation and recorded GRFs for the hop, step, and jump phases respectively (Table 1). Penalties restricting depression to $40 \mathrm{~mm}$ resulted in more realistic forces (Figure 4) with differences of 19.4\%, 17.5\%, and $10.2 \%$ (Table 1). Simulations were shown to best match recorded forces when there were no limitations on the compression of the springs representing the foot-ground interface (Figure 5) with differences of $15.2 \%, 13.2 \%$, and $8.7 \%$ (Table 1). In the unrestricted condition the maximum vertical depressions were $43 \mathrm{~mm}$ at the heel in the hop phase, $56 \mathrm{~mm}$ at the heel in the step phase and $48 \mathrm{~mm}$ at the ball joint in the jump phase. In contrast, horizontal displacements in the unrestricted condition were small in the hop (9 $\mathrm{mm}$ at the ball joint) and step ( $8 \mathrm{~mm}$ at the ball joint) phases in which the forefoot contacted the ground first and large in the jump phase $(29 \mathrm{~mm}$ at the heel) where the heel contacted the ground first (Table 2). 

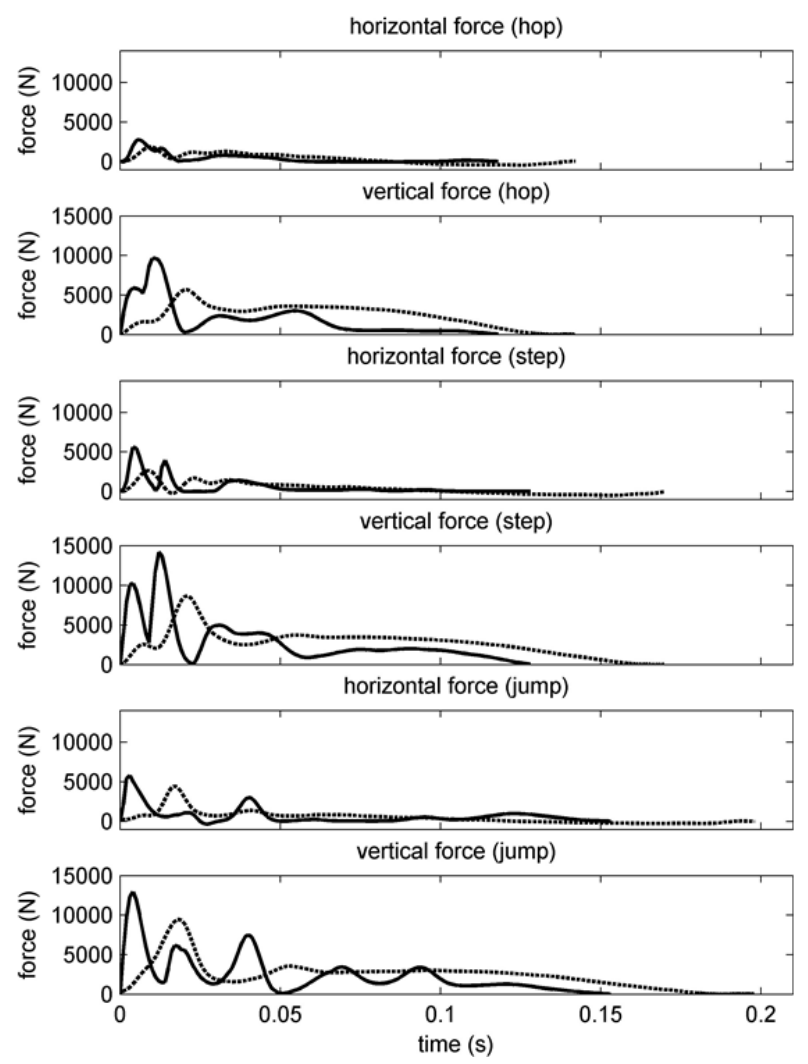

Figure 3. Simulation (solid line) and recorded (broken line) forces for simulations where depression at the foot-ground interface was restricted to $20 \mathrm{~mm}$.
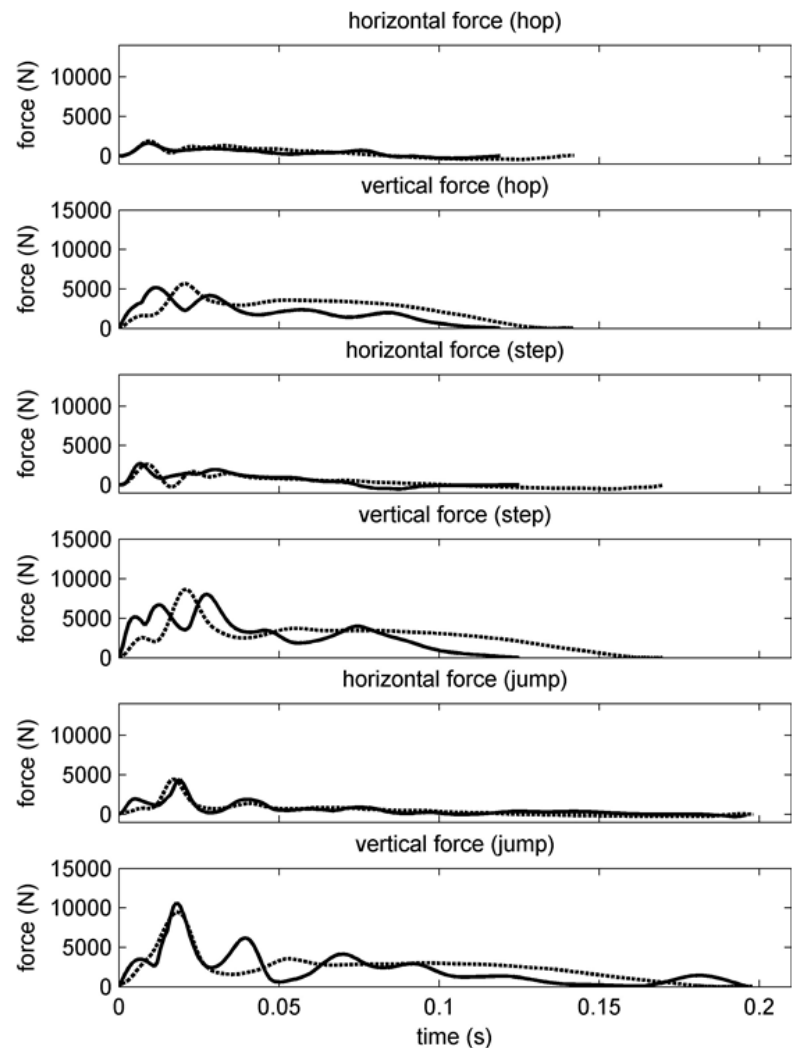

Figure 4. Simulation (solid line) and recorded (broken line) forces for simulations where depression at the foot-ground interface was restricted to $40 \mathrm{~mm}$. 




Figure 5. Simulation (solid line) and recorded (broken line) forces for simulations with no restrictions on depression at the foot-ground interface.

Table 1. Ground reaction force differences for optimisations of each condition and phase

\begin{tabular}{l|l|rrrrrrr}
\hline phase & condition & $\mathrm{fx}(\%)$ & $\mathrm{fz}(\%)$ & $\mathrm{tx}(\%)$ & $\mathrm{tz}(\%)$ & $\mathrm{mx}(\%)$ & $\mathrm{mz}(\%)$ & total(\%) \\
\hline hop & $20 \mathrm{~mm}$ & 27.4 & 44.6 & 33.3 & 45 & 49.8 & 71.3 & 44.3 \\
& $40 \mathrm{~mm}$ & 15.2 & 25.3 & 0 & 40 & 12.8 & 8.5 & 19.4 \\
& unrestricted & 17.1 & 19.7 & 0 & 25 & 4.9 & 6.1 & 15.2 \\
\hline \multirow{2}{*}{ step } & $20 \mathrm{~mm}$ & 33.7 & 34.5 & 55.8 & 43 & 113.3 & 64 & 55.5 \\
& $40 \mathrm{~mm}$ & 17.1 & 21.4 & 22.7 & 28.3 & 2.7 & 7.1 & 17.5 \\
& unrestricted & 14 & 15.7 & 22.7 & 18.8 & 2.4 & 0.5 & 13.2 \\
\hline \multirow{2}{*}{ jump } & $20 \mathrm{~mm}$ & 24.4 & 26.2 & 82.4 & 77.8 & 29.7 & 37.1 & 43.9 \\
& $40 \mathrm{~mm}$ & 9.5 & 15.4 & 11.6 & 0.1 & 1.6 & 12.8 & 10.2 \\
& unrestricted & 9.1 & 14.3 & 0.1 & 5.4 & 3.7 & 2 & 8.7 \\
\hline
\end{tabular}

$f x$ and $f z$ are the RMS differences between the force time histories expressed as a percentage of peak force, $t x$ and $t z$ are the percentage differences in time to peak force, and $m x$ and $m z$ are the percentage differences in the magnitude of peak force

Table 2. Maximum horizontal and vertical spring compression for each condition and each phase of the triple jump

\begin{tabular}{l|l|cc}
\hline phase & condition & $\begin{array}{c}\text { horizontal } \\
\text { compression }(\mathrm{mm})\end{array}$ & $\begin{array}{c}\text { vertical } \\
\text { compression }(\mathrm{mm})\end{array}$ \\
\hline hop & $20 \mathrm{~mm}$ & 12 & 18 \\
& $40 \mathrm{~mm}$ & 20 & 31 \\
& unrestricted & 9 & 43 \\
\hline \multirow{2}{*}{ step } & $20 \mathrm{~mm}$ & 13 & 20 \\
& $40 \mathrm{~mm}$ & 15 & 39 \\
& unrestricted & 8 & 56 \\
\hline jump & $20 \mathrm{~mm}$ & 19 & 20 \\
& $40 \mathrm{~mm}$ & 26 & 36 \\
& unrestricted & 29 & 48 \\
\hline \multicolumn{3}{|c}{}
\end{tabular}


Simulations in which compression in the springs of the foot-ground interface were restricted to $20 \mathrm{~mm}$ showed a trend of greater coefficients governing stiffness and damping in the vertical direction when compared to the $40 \mathrm{~mm}$ and unrestricted conditions, whereas coefficients governing the horizontal springs showed no consistent changes (Tables 3-5). The increased stiffness was manifested in larger force peaks that were reached sooner. The only horizontal limit approached across all optimisations was in the jump phase $(19 \mathrm{~mm})$ in the $20 \mathrm{~mm}$ condition (Table 2). Vertically the limit was hit in the step and jump phases in the $20 \mathrm{~mm}$ condition, and approached in the step phase $(39 \mathrm{~mm}$ ) in the $40 \mathrm{~mm}$ condition (Table 2).

Table 3. Viscoelastic parameters obtained from simulations with spring compression restricted to $20 \mathrm{~mm}$

\begin{tabular}{|c|c|}
\hline parameter & value \\
\hline linear vertical forefoot stiffness $-\mathrm{k}_{11} \& \mathrm{k}_{12}\left(\mathrm{Nm}^{-1}\right)$ & $0.150 \times 10^{6}$ \\
\hline linear vertical heel stiffness $-\mathrm{k}_{13}\left(\mathrm{Nm}^{-1}\right)$ & $0.162 \times 10^{6}$ \\
\hline quadratic vertical forefoot stiffness $-k_{21} \& k_{22}\left(\mathrm{Nm}^{-2}\right)$ & $1.913 \times 10^{6}$ \\
\hline quadratic vertical heel stiffness $-\mathrm{k}_{23}\left(\mathrm{Nm}^{-2}\right)$ & $1.868 \times 10^{6}$ \\
\hline vertical forefoot damping $-\mathrm{k}_{31} \& \mathrm{k}_{32}\left(\mathrm{Nsm}^{-2}\right)$ & $0.125 \times 10^{6}$ \\
\hline vertical heel damping $-\mathrm{k}_{33}\left(\mathrm{Nsm}^{-2}\right)$ & $0.293 \times 10^{6}$ \\
\hline linear horizontal forefoot stiffness $-\mathrm{k}_{41} \& \mathrm{k}_{42}\left(\mathrm{~m}^{-1}\right)^{\star}$ & 8 \\
\hline linear horizontal heel stiffness $-\mathrm{k}_{43}\left(\mathrm{~m}^{-1}\right)^{\star}$ & 1 \\
\hline quadratic horizontal forefoot stiffness $-k_{51} \& k_{52}\left(m^{-2}\right)^{\star}$ & 3539 \\
\hline quadratic horizontal heel stiffness $-k_{53}\left(m^{-2}\right)^{*}$ & 459 \\
\hline horizontal forefoot damping $-k_{61} \& k_{62}\left(s^{-2}\right)^{*}$ & 52 \\
\hline horizontal heel damping $-\mathrm{k}_{63}\left(\mathrm{sm}^{-2}\right)^{*}$ & 100 \\
\hline wobbling mass stiffness shank $-\mathrm{k}_{71}\left(\mathrm{Nm}^{-3}\right)$ & $9.71 \times 10^{6}$ \\
\hline wobbling mass stiffness thigh $-\mathrm{k}_{72}\left(\mathrm{Nm}^{-3}\right)$ & $29.45 \times 10^{6}$ \\
\hline wobbling mass stiffness trunk $-\mathrm{k}_{73}\left(\mathrm{Nm}^{-3}\right)$ & $13.58 \times 10^{6}$ \\
\hline wobbling mass damping shank $-\mathrm{k}_{81}\left(\mathrm{Nsm}^{-1}\right)$ & 337 \\
\hline wobbling mass damping thigh $-\mathrm{k}_{82}\left(\mathrm{Nsm}^{-1}\right)$ & 239 \\
\hline wobbling mass damping trunk $-\mathrm{k}_{83}\left(\mathrm{Nsm}^{-1}\right)$ & 64 \\
\hline
\end{tabular}


Table 4. Viscoelastic parameters obtained from simulations with spring compression restricted to $40 \mathrm{~mm}$

\begin{tabular}{lr}
\hline parameter & \multicolumn{1}{l}{ value } \\
\hline linear vertical forefoot stiffness $-\mathrm{k}_{11} \& \mathrm{k}_{12}\left(\mathrm{Nm}^{-1}\right)$ & $55.96 \times 10^{3}$ \\
linear vertical heel stiffness $-\mathrm{k}_{13}\left(\mathrm{Nm}^{-1}\right)$ & $6.11 \times 10^{3}$ \\
quadratic vertical forefoot stiffness $-\mathrm{k}_{21} \& \mathrm{k}_{22}\left(\mathrm{Nm}^{-2}\right)$ & $1980 \times 10^{3}$ \\
quadratic vertical heel stiffness $-\mathrm{k}_{23}\left(\mathrm{Nm}^{-2}\right)$ & $7.82 \times 10^{3}$ \\
vertical forefoot damping $-\mathrm{k}_{31} \& \mathrm{k}_{32}\left(\mathrm{Nsm}^{-2}\right)$ & $38.32 \times 10^{3}$ \\
vertical heel damping $-\mathrm{k}_{33}\left(\mathrm{Nsm}^{-2}\right)$ & $52.79 \times 10^{3}$ \\
\hline linear horizontal forefoot stiffness $-\mathrm{k}_{41} \& \mathrm{k}_{42}\left(\mathrm{~m}^{-1}\right)^{\star}$ & 8 \\
linear horizontal heel stiffness $-\mathrm{k}_{43}\left(\mathrm{~m}^{-1}\right)^{\star}$ & 3 \\
quadratic horizontal forefoot stiffness $-\mathrm{k}_{51} \& \mathrm{k}_{52}\left(\mathrm{~m}^{-2}\right)^{\star}$ & 2346 \\
quadratic horizontal heel stiffness $-\mathrm{k}_{53}\left(\mathrm{~m}^{-2}\right)^{\star}$ & 166 \\
horizontal forefoot damping $-\mathrm{k}_{61} \& \mathrm{k}_{62}\left(\mathrm{sm}^{-2}\right)^{\star}$ & 28 \\
horizontal heel damping $-\mathrm{k}_{63}\left(\mathrm{sm}^{-2}\right)^{\star}$ & 62 \\
\hline wobbling mass stiffness shank $-\mathrm{k}_{71}\left(\mathrm{Nm}^{-3}\right)$ & $30.23 \times 10^{6}$ \\
wobbling mass stiffness thigh $-\mathrm{k}_{72}\left(\mathrm{Nm}^{-3}\right)$ & $7.10 \times 10^{6}$ \\
wobbling mass stiffness trunk $-\mathrm{k}_{73}\left(\mathrm{Nm}^{-3}\right)$ & $28.48 \times 10^{6}$ \\
wobbling mass damping shank $-\mathrm{k}_{81}\left(\mathrm{Nsm}^{-1}\right)$ & 24 \\
wobbling mass damping thigh $-\mathrm{k}_{82}\left(\mathrm{Nsm}^{-1}\right)$ & 298 \\
\hline wobbling mass damping trunk $-\mathrm{k}_{83}\left(\mathrm{Nsm}^{-1}\right)$ & 288 \\
\hline
\end{tabular}

* horizontal coefficients have different dimensions since horizontal force is a function of vertical force 
Table 5. Viscoelastic parameters obtained from simulations with no restrictions on spring compression

\begin{tabular}{|c|c|}
\hline parameter & value \\
\hline linear vertical forefoot stiffness $-\mathrm{k}_{11} \& \mathrm{k}_{12}\left(\mathrm{Nm}^{-1}\right)$ & $52.05 \times 10^{3}$ \\
\hline linear vertical heel stiffness $-\mathrm{k}_{13}\left(\mathrm{Nm}^{-1}\right)$ & $7.09 \times 10^{3}$ \\
\hline quadratic vertical forefoot stiffness $-\mathrm{k}_{21} \& \mathrm{k}_{22}\left(\mathrm{Nm}^{-2}\right)$ & $15.64 \times 10^{3}$ \\
\hline quadratic vertical heel stiffness $-\mathrm{k}_{23}\left(\mathrm{Nm}^{-2}\right)$ & $414.47 \times 10^{3}$ \\
\hline vertical forefoot damping $-\mathrm{k}_{31} \& \mathrm{k}_{32}\left(\mathrm{Nsm}^{-2}\right)$ & $18.77 \times 10^{3}$ \\
\hline vertical heel damping $-\mathrm{k}_{33}\left(\mathrm{Nsm}^{-2}\right)$ & $7.82 \times 10^{3}$ \\
\hline linear horizontal forefoot stiffness $-\mathrm{k}_{41} \& \mathrm{k}_{42}\left(\mathrm{~m}^{-1}\right)^{\star}$ & 32 \\
\hline linear horizontal heel stiffness $-k_{43}\left(m^{-1}\right)^{\star}$ & 1 \\
\hline quadratic horizontal forefoot stiffness $-k_{51} \& k_{52}\left(m^{-2}\right)^{\star}$ & 7126 \\
\hline quadratic horizontal heel stiffness $-k_{53}\left(m^{-2}\right)^{\star}$ & 753 \\
\hline horizontal forefoot damping $-\mathrm{k}_{61} \& \mathrm{k}_{62}\left(\mathrm{sm}^{-2}\right)^{\star}$ & 48 \\
\hline horizontal heel damping $-\mathrm{k}_{63}\left(\mathrm{sm}^{-2}\right)^{*}$ & 27 \\
\hline wobbling mass stiffness shank $-\mathrm{k}_{71}\left(\mathrm{Nm}^{-3}\right)$ & $95.94 \times 10^{6}$ \\
\hline wobbling mass stiffness thigh $-\mathrm{k}_{72}\left(\mathrm{Nm}^{-3}\right)$ & $6.32 \times 10^{6}$ \\
\hline wobbling mass stiffness trunk $-\mathrm{k}_{73}\left(\mathrm{Nm}^{-3}\right)$ & $36.52 \times 10^{6}$ \\
\hline wobbling mass damping shank $-\mathrm{k}_{81}\left(\mathrm{Nsm}^{-1}\right)$ & 293 \\
\hline wobbling mass damping thigh $-\mathrm{k}_{82}\left(\mathrm{Nsm}^{-1}\right)$ & 362 \\
\hline wobbling mass damping trunk $-\mathrm{k}_{83}\left(\mathrm{Nsm}^{-1}\right)$ & 3089 \\
\hline
\end{tabular}

Coefficients governing the stiffness of the wobbling masses showed a decrease from no restriction to the $20 \mathrm{~mm}$ restriction condition in the shank and trunk, with an increase in thigh stiffness (Tables 3-5). Damping values for the trunk showed the opposite trend to the stiffness values; increasing from the $20 \mathrm{~mm}$ restriction to the unrestricted condition. The reductions in stiffness led to increased displacement of the large mass representing the soft tissue of the trunk in the $20 \mathrm{~mm}$ condition (Figures 6-7). However there were no consistent trends in wobbling mass displacement with increasing restrictions on spring compression. 

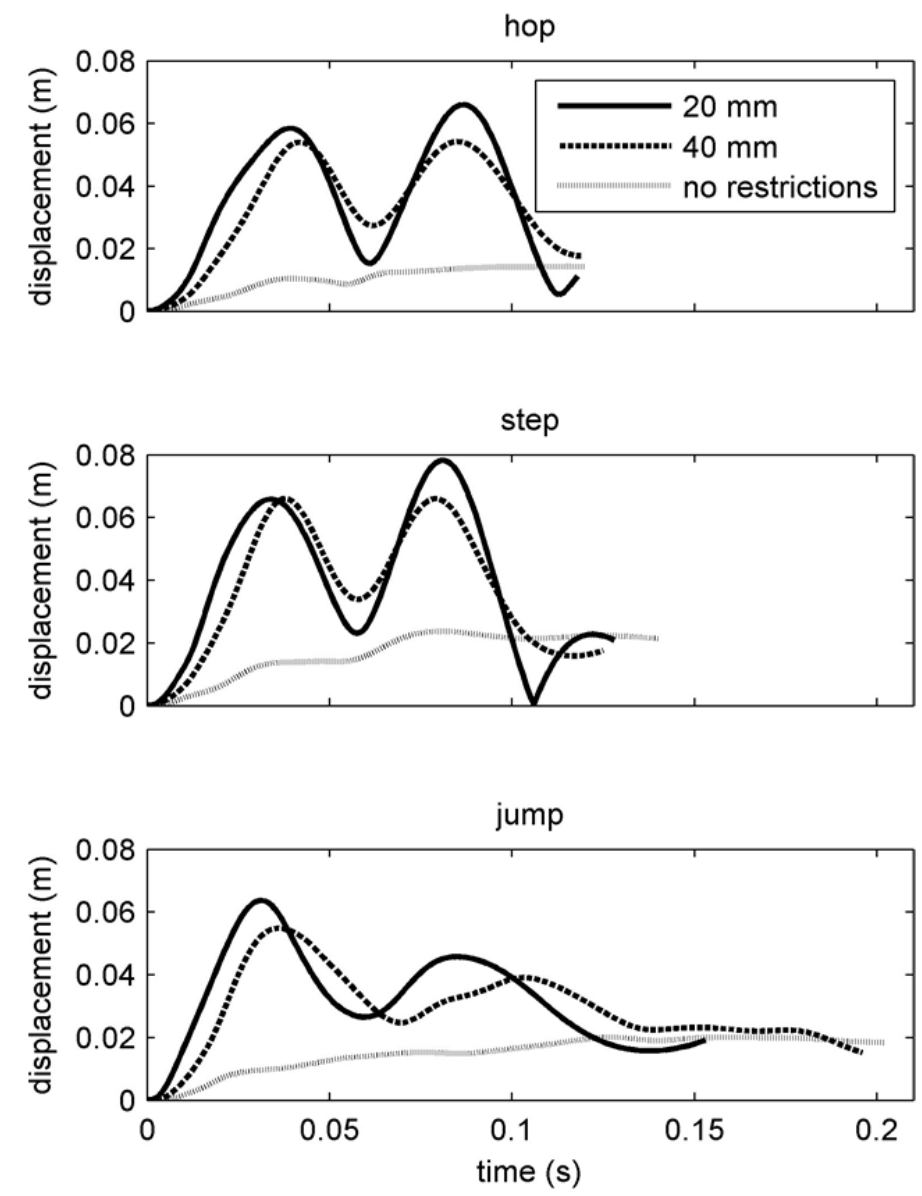

Figure 6. Displacement of trunk wobbling mass in each phase of the triple jump and in each condition.

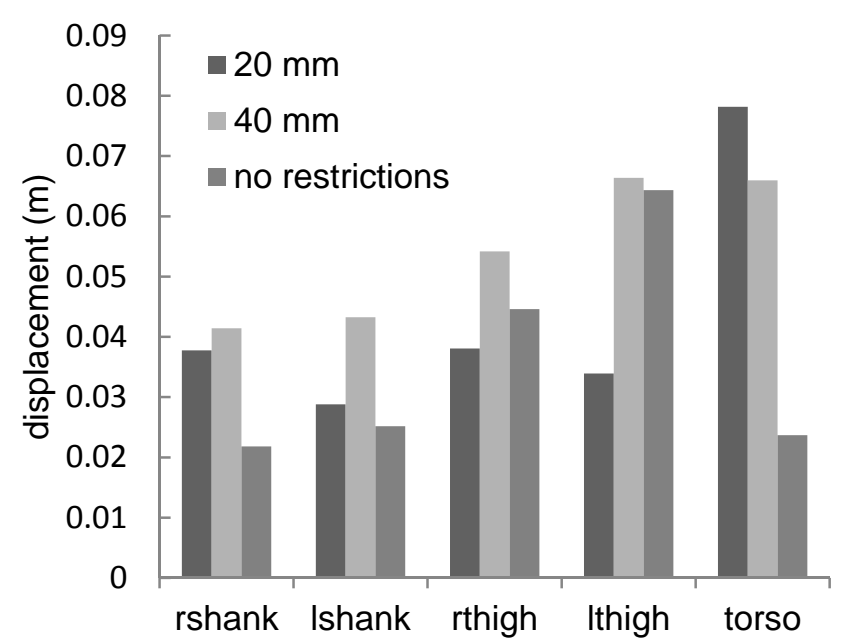

Figure 7. Maximum displacements of wobbling masses in each phase of the triple jump and in each condition.

Ground contact time in the unrestricted condition was the longest in each phase, with simulation values tending to underestimate ground contact time in all conditions for the first two phases (Table 6). With one exception there was a trend towards increased ground contact times with increased bounds on spring compression, with the unrestricted condition being closest to recorded measures overall. 
Table 6. Ground contact time in each condition and each phase versus measured performance

\begin{tabular}{l|l|c}
\hline phase & condition & time $(\mathrm{ms})$ \\
\hline hop & $20 \mathrm{~mm}$ & 118 \\
& $40 \mathrm{~mm}$ & 119 \\
& unrestricted & 120 \\
& performance & 142 \\
\hline \multirow{2}{*}{ step } & $20 \mathrm{~mm}$ & 128 \\
& $40 \mathrm{~mm}$ & 125 \\
& unrestricted & 140 \\
& performance & 170 \\
\hline \multirow{2}{*}{ jump } & $20 \mathrm{~mm}$ & 153 \\
& $40 \mathrm{~mm}$ & 196 \\
& unrestricted & 202 \\
& performance & 198 \\
\hline
\end{tabular}

The RMS differences in trunk angle between simulation in the unrestricted condition and recorded performance were $0.6^{\circ}, 0.7^{\circ}$ and $0.8^{\circ}$ in the hop, step and jump phases respectively. The differences in vertical mass centre positions at the time of maximum depression in the unrestricted condition were $2 \mathrm{~mm}, 4 \mathrm{~mm}$ and 4 $\mathrm{mm}$ in the hop, step and jump phases respectively.

\section{Discussion}

When high levels of compliance were allowed at the foot-ground interface, the pin-linked simulation model was able to match recorded GRFs well during an extremely high impact activity (Figure 5). The unrestricted condition also led to ground contact times that best matched the performance measures, with the $20 \mathrm{~mm}$ condition underestimating ground contact times by the greatest amount in each phase. The trunk angle in simulations lay within $1^{\circ}$ (RMS) of recorded values indicating that the point of application of vertical force on the foot was accurate. The viscoelastic parameters were determined using all three ground contact phases of the triple jump in order that it was robust to changes in kinematics. This method has previously been shown to yield parameters that accurately reproduce performance measures in a separate trial by the same subject (Yeadon et al., 2006). These results indicate that a pin-linked subject-specific simulation model would be sufficient for simulations aiming to match or optimise sporting technique, since GRF time histories are realistic and therefore performance variables such as net impulse and takeoff velocity will be realistic.

In order to determine how closely the displacement of the whole body COM in the unrestricted simulation matched that of the recorded data, the recorded vertical GRF was used to double integrate the calculated acceleration to give the vertical position of the COM over time. In each phase the position of the COM at the time of peak depression in the simulation differed by less than $4 \mathrm{~mm}$ between simulation and recorded data. Thus the $\mathrm{COM}$ in the recorded performance descended to a comparable position as in the simulations for which the foot springs compressed more than $40 \mathrm{~mm}$. As a consequence there must be additional compliance in the real system beyond the $20 \mathrm{~mm}$ compression at the foot-ground interface. It is hypothesised that the high level of compliance in the foot springs of the model in the unrestricted case (>40 mm) is a consequence of not modelling compliance in the joints and bones of the legs and trunk.

The model was not able to reproduce accurate GRFs when it was limited to realistic depressions $(20 \mathrm{~mm})$ at the foot-ground interface. This is despite the 
inclusion of wobbling masses representing the soft tissue of the legs and trunk. The wobbling masses accounted for approximately two thirds of the total mass of the model, and so movement of this mass might have been expected to provide sufficient compliance in the system. However the fact that there was no consistent trend in the parameters and displacement of the wobbling masses between the different conditions, and that no displacement bounds were reached during optimisation (Figure 7), indicates that the inability of the model to reproduce accurate GRFs in the $20 \mathrm{~mm}$ condition was not a result of excessive strictures on wobbling mass displacement. The fact that accurate GRFs could not be reproduced in this condition, despite the inclusion of wobbling masses in the model, indicates that other compliance in the human body will need to be represented if models are to accurately reproduce GRFs with realistic levels of depression at the foot-ground interface in the future.

Mills et al. (2010) found extremely high bending moments of $377 \mathrm{Nm}$ and 266 $\mathrm{Nm}$ at the thigh and shank respectively in simulations of gymnastics landings. The former value exceeded the maximum bending moment of the human femoral bones measured in vitro by Martens et al. (1986), which resulted in displacements of $17 \mathrm{~mm}$ of the ends of the bone relative to the centre. Mills et al. stated that they used bone bending moments only as an indicator of relative injury risk, and the high reported values are likely to be a result of their model not incorporating the effects of muscles in mitigating bone bending moments (Duda et al., 1997). Despite this, bone bending may make up a non-negligible component of the force-attenuating properties of the body during extremely high loading. Rostedt et al. (1998) investigated the axial stiffness of functional spinal units (FSUs) from the lumbar region. Although obtained from a lower range of loading levels, the stiffness relationship they observed predicts that, in order to compress the unit by $1 \mathrm{~mm}$, a load of approximately $3500 \mathrm{~N}$ would have to be applied. The high forces endured during triple jumping mean this level of compression is not out of the question, and an average of $1 \mathrm{~mm}$ compression in the lumbar and thoracic vertebrae could lead to overall spinal compressions of $16 \mathrm{~mm}$. In addition to this, the joints of the leg also greatly attenuate forces: e.g. Hoshino and Wallace (1987) found an 80\% increase in peak transmitted force through knee joints with knee replacements when compared to intact knee joints. It has been shown that higher frequency accelerations (greater than $6 \mathrm{~Hz}$ ) are attenuated by the body and are not transmitted to the head during running, irrespective of the magnitude of the shock measured at the shank (Shorten and Winslow, 1992). The sum total of all the force attenuating properties in the skeletal system is undoubtedly high whereas in rigid pin-linked simulation models accelerations are transmitted instantaneously and undamped through the rigid link system.

A number of choices were made in how various aspects were modelled. The simulation model employed a single segment to represent the trunk whereas it might be expected that having an articulated spine could improve the biofidelity of the model. However unless joint compliance were to be introduced into the representation of the spine, the vertical movement of the mass centre would not be replicated in a model with realistic foot compression. Quadratic stiffness was used at the foot-ground interface since it gave a better match than linear stiffness. The horizontal force was expressed as a function of the vertical force, in order that it would decay to zero with the vertical force. This gave better results than making the horizontal force a function of the vertical displacement. Three foot contact points were used since the foot was modelled as two segments. This allowed the resultant vertical force to act at an appropriate point between heel and toe. Increasing the 
sophistication of the modelling choices would not have changed the conclusions of this study.

It has been shown that a pin-linked simulation model can reproduce realistic GRFs if additional compliance is allowed at the foot-ground interface, therefore future simulation studies of sporting performance should allow a suitably high level of compliance in the springs of the feet in order that accurate GRFs can be obtained. On the other hand a lack of compliance in joints and bones will render a model unsuitable for investigations into internal joint loading. In such investigations force attenuating properties need to be incorporated into the link structure of the simulation model.

\section{References}

Alexander, R. M., 1990. Optimum take-off techniques for high and long jumps. Philosophical Transactions of the Royal Society of London: Series B 329, 3-10.

Alexander, R.M., Bennett, M., 1989. How elastic is a running shoe? New Scientist, 15 July, 45-46.

Allen, S. J., King, M. A., Yeadon, M. R., 2010. Is a single or double arm technique more advantageous in triple jumping? Journal of Biomechanics 43, 3156-3161.

Anderson, F.C., Pandy, M.G., 2001. Dynamic optimization of human walking. Journal of Biomechanical Engineering 123, 381-390.

Carroll, D.L., 1996. Chemical laser modeling with genetic algorithms, AIAA Journal 34, 338-346.

Chu, M. L., Yazdani-Ardakani, S., Gradisar, I. A., Askew, M. J., 1986. An in vitro simulation study of impulsive force transmission along the lower skeletal extremity. Journal of Biomechanics 19, 979-981, 983-987.

Duda, G.N., Schneider, E. and Chao, E.Y.S., 1997. Internal forces and moments in the femur during walking. Journal of Biomechanics 30, 933-941.

Gruber, K., Ruder, H., Denoth, J., Schneider, K., 1998. A comparative study of impact dynamics: wobbling mass model versus rigid body models. Journal of Biomechanics 31, 439-444.

Hay, J.G., 1993. Citius, altius, longius (faster, higher, longer): the biomechanics of jumping for distance. Journal of Biomechanics 26, 7-21.

Hoshino, A., Wallace, W.A., 1987. Impact-absorbing properties of the human knee. Journal of Bone and Joint Surgery - British Volume 69, 807-811.

Kane, T.R., Levinson, D.A., 1996. Dynamics online: theory and implementations with AUTOLEV. First edn. Sunnyvale: Online Dynamics Inc.

King, M.A., Wilson, C., Yeadon, M.R., 2006. Evaluation of a torque-driven model of jumping for height. Journal of Applied Biomechanics 22, 264-274.

Lafortune, M.A., Lambert, C., Lake, M., 1992. Skin marker displacement at the knee joint. In Proceedings of the Second North American Congress on Biomechanics, Chicago, IL, USA.

Martens, M., van Audekercke, R., de Meester, P., Mulier, J. C., 1986. Mechanical behaviour of femoral bones in bending loading. Journal of Biomechanics 19, 443-454.

Mills, C., Pain, M.T.G., Yeadon, M.R., 2010. Modifying landing mat properties may decrease peak contact forces but increase forefoot forces in gymnastics landings. Sports Biomechanics 9, 153-164. 
Minetti, A.E., Belli, G., 1994. A model for the estimation of visceral mass displacement in periodic movements, Journal of Biomechanics 27, 97-101.

Neptune, R.R., Wright, I.C., Bogert, A.J. van den, 2000. A method for numerical simulation of single limb ground contact events: application to heel-toe running. Journal of Computer Methods in Biomechanics and Biomedical Engineering 3, 321-334.

Otten, E., 2003. Inverse and forward dynamics: models of multi-body systems. Philosophical Transactions of the Royal Society B 358, 1493-1500.

Pain, M.T.G., Challis, J.H., 2001. The role of the heel pad and shank soft tissue during impacts: a further resolution of a paradox. Journal of Biomechanics 34, 327-333.

Pain, M. T. G., Challis, J. H., 2004. Wobbling mass influence on impact ground reaction forces: a simulation model sensitivity analysis. Journal of Applied Biomechanics 20, 309-316.

Perttunen, J., Kyrolainen, H., Komi, P.V., Heinonen, A. 2000. Biomechanical loading in the triple jump. Journal of Sports Sciences 18, 363-370.

Radin, E.L., Paul, I.L., 1970. Does cartilage compliance reduce skeletal impact loads? Relative force attenuating properties of articular cartilage, synovial fluid, periarticular soft tissues and bones. Arthritis and Rheumatism 13, 139-144.

Rostedt, M., Ekstrom, L., Broman, H., Hansson, T., 1998. Axial stiffness of human lumbar motion segments, force dependence. Journal of Biomechanics 31, 503509.

Seyfarth, A., 2000. Elastically operating legs - strategies and construction principles. $P h D$ thesis, Jena University, Germany.

Shorten, M.R., Winslow, D.S., 1992. Spectral analysis of impact shock during running. International Journal of Sports Biomechanics 8, 288-304.

van Soest, A. J., Schwab, A. L., Bobbert, M. F., van Ingen Schenau, G. J., 1993. The influence of the biarticularity of the gastrocnemius muscle on vertical-jumping achievement. Journal of Biomechanics 26, 1-8.

Wilson, C., King, M.A., Yeadon, M.R., 2006. Determination of subject-specific model parameters for visco-elastic elements. Journal of Biomechanics 39, 1883-1890.

Wood, G.A., Jennings, L.S., 1979. On the use of spline functions in data smoothing. Journal of Biomechanics 12, 477-479.

Yeadon, M.R., 1990. The simulation of aerial movement - II. A mathematical inertia model of the human body. Journal of Biomechanics 23, 67-74.

Yeadon, M.R. and King, M.A., 2002. Evaluation of a torque-driven simulation model of tumbling. Journal of Applied Biomechanics 18, 195-206.

Yeadon, M.R., Kong, P.W., King, M.A., 2006. Parameter determination for a computer simulation model of a diver and a springboard. Journal of Applied Biomechanics 22, 167-176. 